\title{
Tests of the mode-coupling theory for a molten salt
}

\author{
By M. Fuchs†, H. Z. Cummins $\ddagger$, W. M., Du $\ddagger$, W. Gotze $\dagger$, \\ A. LATZ†\&, G. LI $\ddagger$ and N. J. TAO $\$$ I \\ † Physik-Department, Technische Universität Munchen, \\ D-85748 Garching, Germany \\ $\ddagger$ City College of The City University of New York, \\ New York, NY 10031, USA
}

\begin{abstract}
A recently reperted analysis (Cummins et al., 1993, Phys. Rev. E, 47, 4223) of depolarized light and neutron scattering experiments in $\mathrm{CaKNO}_{3}$ using the mode-coupling theory is extended. The temperature dependence of the critical amplitudes found in this analysis is explained.
\end{abstract}

\section{§1. INTRODUCTION}

Undercooled liquids exhibit slow, temperature-sensitive and non-exponentional relaxation processes. It is of interest whether the mode coupling theory (MCT) of the liquid-to-glass transition can qualitatively and quantitatively account for this anomalous relaxation behaviour; see Ngai (1994) for an overview of recent theoretical and experimental work. Experiments on the well-studied glass-forming ionic salt, calcium potassium nitrate $\left(\mathrm{CKN}, \mathrm{Ca}_{0.4} \mathrm{~K}_{0.6}\left(\mathrm{NO}_{3}\right)_{1.4}\right)$, both by neutron spin-echo (Mezei 1991) and by depolarized light scattering ( $\mathrm{Li}, \mathrm{Du}$, Chen, Cummins and Tao 1992a) have been analysed in order to test predictions of the MCT. In this paper the analysis presented in Cummins et al. (1993) is summarized and the question of the temperature dependence of the so-called critical amplitudes $h_{\mathrm{ls}}$ and $h_{\mathrm{n}}$ (see fig. $2(b)$ of Cummins et al. (1993)) is studied further. The temperature dependence of $h_{\mathrm{is}}$ and $h_{\mathrm{n}}$ found in Cummins et al. (1993) constituted the only qualitative deviation from the asymptotic MCT results.

\section{$\S 2$, SUMMARY OF SOME MODE COUPLING THEORY RESULTS}

The MCT considers a closed system of equations of motion for density fluctuation functions $\Phi_{\mathrm{q}}(t)=\left\langle\rho_{\mathrm{q}}^{*}(t) \rho_{\mathrm{q}}\right\rangle /\left\langle\left.\rho_{\mathrm{a}}\right|^{2}\right\rangle$; see Götze (1991) and Götze and Sjögren (1992) for further information. Within an intermediate time window, the full equations are solved by the following factorization of time and wave-vector dependencies (Götze and Sjögren 1987):

$$
\Phi_{\mathrm{q}}(t)=f_{\mathrm{q}}^{\mathrm{c}}+h_{\mathrm{q}} G\left(t, \sigma, \delta t_{0}\right) \quad \text { for }|G| \ll 1 .
$$

The requirement $|G| \ll 1$ defines the time window $t_{0} \ll t \ll \tau_{\alpha}$, where eqn. (1) holds; within the MCT it is called the $\beta$-relaxation regime, $t_{0}$ denotes a typical microscopic time scale $\left(t_{0} \approx 1 \mathrm{ps}\right.$ ) and $\tau_{\alpha}$ the $\alpha$-relaxation time, which exceeds $100 \mathrm{~s}$ at the calorimetric glass USA.

\$ Present address: Department of Physics, Florida International University, Miami, FL 33199 ,

Tresent address: Institute for Physical Science, University of Maryland, College Park, MD 20742, USA.

||Also at: Max Planck Institut für Physik (Wemer Heisenberg Institut), D80805 München, Germany. 
transition temperature $T_{\mathrm{g}}$. The $\alpha$-relaxation strength $f_{\mathrm{q}}^{c}$ and the critical amplitude $h_{\mathrm{q}}$ can in principle be calculated from the equilibrium static structure factor $S_{\mathrm{q}}\left(T_{\mathrm{c}}\right)$ taken at the critical temperature $T_{\mathrm{c}}, T_{\mathrm{c}}$ is defined by zero. $\sigma\left(T_{\mathrm{c}}\right)=0$, of the separation parameter $\sigma(T)$, which in turn can be calculated in principle from $S_{\mathrm{q}}(T)$. In Fuchs, Hofacker and Latz (1992c) these quantities were calculated for a liquid of hard spheres and in van Megen and Underwood (1993a, b) they were compared with experiments. For the complex glass former CKN, the parameters entering eqn. (1) have to be treated as fit parameters. The rate $\delta$, often called the hopping rate, is expected to show an Arrhenius temperature dependence $\delta \propto \exp (-E / k T)$ (Sjögren 1990); see Götze and Sjögren (1987) for a detailed expression for $\delta$. The decoupling of temporal and spatial variations expressed in eqn. (1) identifies the $\beta$-dynamics as being localized. This is further supported by the calculation of the radial-dependent critical amplitude $h_{\mathrm{r}}$ for hard spheres which turns out to be short ranged (Barrat, Götze and Latz 1989).

The $\beta$-correlator $G(t)$ and the corresponding dynamical susceptibility $\chi_{q}^{\prime \prime}(\omega)=h_{q} \omega \int_{0}^{\infty} \mathrm{d} t \cos (\omega t) G(t)$ are determined by the following equation (Götze and Sjögren 1987):

$$
\sigma-\delta t+2 G^{2}(t)=\frac{\mathrm{d}}{\mathrm{d} t} \int_{0}^{t} \mathrm{~d} t^{\prime} G\left(t-t^{\prime}\right) G\left(t^{\prime}\right),
$$

where the exponent parameter $\lambda$ is a functional of the equilibrium static structure factor $S_{\mathrm{q}}\left(T_{\mathrm{c}}\right)$ at the critical temperature $T_{\mathrm{c}}$.

If the two temperature-dependent control parameters $\left(\sigma(T), \delta(T) t_{0}\right)$ are small, the time window where eqn. (1) holds increases and for $\left(\sigma, \delta t_{0}\right)=(0,0)$ it extends to infinity. This slowing down of the dynamics in eqn. (2) can most easily be seen from the two-parameter scaling law which $G$ and consecutively $\chi^{\prime \prime}$ obey (Götze and Sjögren 1987)

$$
\begin{aligned}
G(t, \sigma, \delta) & =\Omega^{a} \hat{G}\left(t \Omega / t_{0}, \sigma / \Omega^{2 a}, \delta t_{0} / \Omega^{2 a+1}\right) \\
\chi^{\prime \prime}(\omega, \sigma, \delta) & =\Omega^{a} \hat{\chi}^{\prime \prime}\left(\omega t_{0} / \Omega, \sigma / \Omega^{2 a}, \delta t_{0} / \Omega^{2 a+1}\right)
\end{aligned} \quad \text { for arbitrary } \Omega>0 .
$$

If the function $\hat{G}(t, \hat{\sigma}, \hat{\delta})$ is known for the parameter point $(\hat{\sigma}), \hat{\delta})$, then for all parameters on this scaling line parameterized by $\Omega,\left(\sigma, \delta t_{0}\right)=\left(\Omega^{2 a} \hat{\sigma}, \Omega^{(2 a+1)} \hat{\delta} t_{0}\right)$, the correlator $G(t, \sigma, \delta)$ transforms self-similarly: $G(t, \sigma, \delta)=\Omega^{a} \hat{G}\left(t \Omega / t_{0}, \hat{\sigma}, \hat{\delta}\right)$. If the parameters move on this scaling line according to $\Omega \rightarrow 0$, then dynamical features of $G$ move to longer times.

The MCT offers this slowing down as an explanation for slow relaxation phenomena in an intermediate frequency window below the microscopic excitations of a liquid if $\left(\sigma, \delta t_{0}\right)$ are small. Experiments on complex glass formers can test these predictions because of the factorization property (1) which states that, in the $\beta$-relaxation region, different density susceptibilities $\chi_{q}^{\prime \prime}(\omega, \sigma, \delta)$ at the same temperature only differ by constant factors $h_{\mathrm{q}}$. Moreover, the $\beta$-correlator is determined by the single material-dependent parameter $\lambda$, which allows severe tests if the master functions $\hat{G}$ or $\hat{\chi}^{\prime \prime}$ have been extracted. Corrections to eqn. (1) vanish if $\left(\sigma, \delta t_{0}\right)$ become smaller (Fuchs, Götze, Hildebrand and Latz 1992a). An important test of eqns. (1) and (2) therefore is to demonstrate that their range of validity expands if $\left(\sigma, \delta t_{0}\right)$ approach $(0,0)$, i.e. for temperatures $T$ approaching $T_{c}$.

At the critical point $\left(\sigma, \delta t_{0}\right)=(0,0)$, eqn. (2) is solved by the so-called critical law (Götze 1991) $G(t, 0,0)=\left(t / t_{0}\right)^{-a}$, where $0<a<0.4$ and $t_{0}$ has to be found by matching to the transient microscopic dynamics. Finite control parameters $\left(\sigma, \delta t_{0}\right)$ introduce scaling times $t_{\mathrm{sc}}^{\beta}\left(\sigma, \delta t_{0}\right)$ where the critical law is cut off and $G$ crosses over to different $t$ behaviours. A complete discussion of $G$ and eqn. (2) can be found in Fuchs $e t$ al. (1992a). In the following only some asymptotes of $\chi^{\prime \prime}(\omega, \sigma, \delta)$ will be given in order to show the qualitative 
behaviour of the numerical solutions of eqn. (2), which are relevant to the experiments discussed later on.

In the so-called idealized MCT, $\delta=0$ is assumed. A single $\beta$-time scale $t_{\mathrm{sc}}^{\beta}(\sigma, 0)=t_{\sigma}$ is obtained: $t_{\sigma}=\omega_{\sigma}^{-1}=t_{0} \sigma^{-1 / 2 a}$. Two cases, $\sigma>0$ and $\sigma<0$, have to be considered separately in the dynamical susceptibility $\chi^{\prime \prime}(\omega, \sigma, 0)$. For $\sigma>0\left(T<T_{\mathrm{c}}\right), \chi^{\prime \prime}$ crosses over from the critical law to a regular frequency behaviour. This leads to a knee in the $\log \chi^{\prime \prime}$ versus $\log \omega$ curve located close to the crossover time $\omega_{\sigma}$.

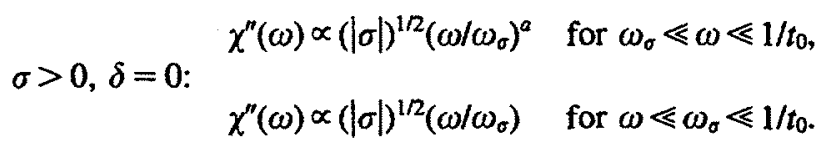

For $\sigma<0\left(T>T_{\mathrm{c}}\right), G$ crosses over to the von Schweidler decay $G\left(t \gg t_{\sigma}\right) \rightarrow-(|\sigma|)^{1 / 2} B\left(t / t_{\sigma}\right)^{b}$. This leads to a minimum in the susceptibility situated around $\omega_{\sigma}$ :

$$
\begin{aligned}
& \sigma<0, \delta=0 \text { : } \\
& \chi^{\prime \prime}(\omega) \propto(|\sigma|)^{1 / 2}\left(\omega / \omega_{\sigma}\right)^{a} \quad \text { for } 1 / \tau \ll \omega_{\sigma} \ll \omega \ll 1 / t_{0}, \\
& \chi^{\prime \prime}(\omega) \propto(|\sigma|)^{1 / 2}\left(\omega / \omega_{\sigma}\right)^{-b} \text { for } 1 / \tau \ll \omega \ll \omega_{\sigma} \ll 1 / t_{0} .
\end{aligned}
$$

It has to be stressed that the crossover region around $\omega_{\sigma}$ is rather broad. It is therefore often impossible to fit the asymptotic laws (4) directly to the data. In general, fits require the use of the complete $\beta$-susceptibility $\chi^{\prime \prime}(\omega)$ for frequencies not well separated from, $\omega_{\sigma}$ (Götze 1990).

Within the idealized MCT, i.e. neglecting thermally activated transport, the corrections of the slower $\alpha$-process to the above results can be expressed as a second scaling law, which holds close to and above $T_{\mathrm{c}}$ (Götze 1991)

$$
\Phi_{\mathrm{q}}(t)=f_{\mathrm{q}}^{\mathrm{c}} F_{\mathrm{q}}(t / \tau) \quad \text { for } t, \tau \gg t_{\sigma} \text { and } \sigma>0, \delta=0 \text {. }
$$

The normalized $\alpha$-correlators, $F_{\mathrm{q}}$, obey an integro-differential equation which is determined by $S_{\mathrm{q}}\left(T_{\mathrm{c}}\right)$. They are generally well approximated by Kohlrausch functions $F_{\mathrm{q}}(x) \approx \exp \left[-\left(x / x_{\mathrm{q}}\right)^{\beta_{\mathrm{q}}}\right]$ with $T$-independent coefficients $x_{\mathrm{q}}$ and Kohlrausch exponents $\beta_{\mathrm{q}}<1$ (Fuchs 1994). The von Schweidler, law $F_{\mathrm{q}}-1 \propto-(t / \tau)^{b}$ for $t / \tau \rightarrow 0$, is shared by both $\beta$ - and $\alpha$-processes, thereby connecting the two time scales $t_{\sigma}$ and $\tau$

$$
\tau=(-\sigma)^{-1 / 2 b_{\sigma}}=t_{0}|\sigma|^{-1 / 2 a-1 / 2 b} .
$$

In the extended MCT, where $\delta$ is finite and positive, the effects of $\delta$ first appear for long times or small frequencies as can be expected from eqn. (2). Moreover, $\delta(T)$ introduces a natural scale $\sigma_{0}$ for $\sigma$

$$
\sigma_{0}=\left(\delta t_{0}\right)^{2 \alpha / 1+2 a}, \delta>0 .
$$

In the idealized MCT only $|\sigma| \ll 1$ was required to derive the results of eqns. (4). For non-zero $\delta t_{0}$, formulate $(4 b)$ apply for $-1 \ll \sigma \ll-\sigma_{0}$, which entails the condition $\omega \gg \omega_{\delta}=1 / t_{0}\left(\delta t_{0}\right)^{1 / 1+2 a}$. The results $(4 a)$ apply for $1 \gg \sigma \gg \sigma_{0}$, which leads to the condition $\omega \gg \omega_{\mathrm{g}}=\left(\omega_{\sigma} \delta /|\sigma|\right)^{1 / 2}$ (Fuchs et al. 1992a). In the transition region $|\sigma|<\sigma_{0}$ close to $T_{c}$, the critical decay $G \propto t^{-a}$ crosses over at times of the order of $t_{\delta}$, causing a steeper susceptibility minimum situated at $\omega_{\delta}$; for $\lambda \geqslant \pi / 4$ the asymptotes are (Fuchs et al. 1992a)

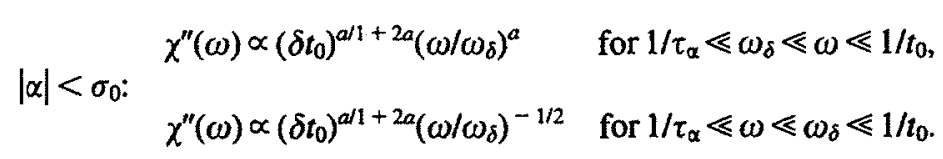

Thereby the result of a vanishing minimum frequency $\omega_{\min }$ for $T \backslash T_{\mathrm{c}}$ implied by eqn. (4b) 
is cut off in the extended MCT, where $\omega_{\min }$ arrests at $\omega_{\delta}$ for $T=T_{\mathrm{c}}$. In the glass region $\sigma>\sigma_{0}$, where the idealized MCT finds no minimum in the $\beta$-relaxation regime, a finite hopping rate, $\delta t_{0}>0$, leads to a steep minimum situated at $\omega_{\mathrm{g}}=\left(\omega_{\sigma} \delta /|\sigma|\right)^{1 / 2}$ which interpolates between the following asymptotes:

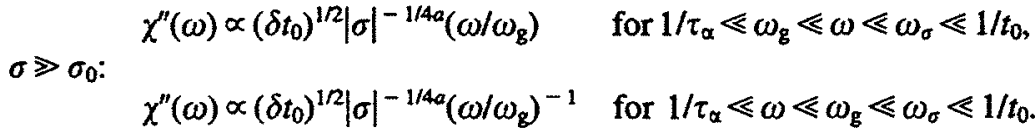

\section{§3. OVERVIEW OF TWO EXPERIMENTS ON CKN}

Depolarized light scattering data in $\mathrm{CKN}$ ( $\mathrm{Li}$ et al. 1992a) were analysed using the susceptibilities obtained from numerical solutions of eqn. (2) (Cummins et al. 1993). The data and corresponding fits are reproduced in fig. 1 . The data show some inelastic Raman contribution above $c .3 \mathrm{THz}$. It is caused by the microscopic transient motion which is not included in the above theoretical results. The slow glassy dynamics leads to a characteristic strong enhancement of the spectra above the expected linear low-frequency asymptote of this microscopic excitation. The Markovian result $\chi^{\prime \prime}\left(\omega t_{0} \ll 1\right) \propto \omega$ is indicated by a dashed line in fig. 1. The final $\alpha$-relaxation peak moves out of the spectral range below $T=140^{\circ} \mathrm{C}$. It is discussed in $\mathrm{Li}$ et al. (1992a) and obeys the scaling law (5) with Kohlrausch exponent $\beta_{\mathrm{ls}}=0.55$ up to the highest temperature studied $\left(T=195^{\circ} \mathrm{C}\right)$.

Fig. 1

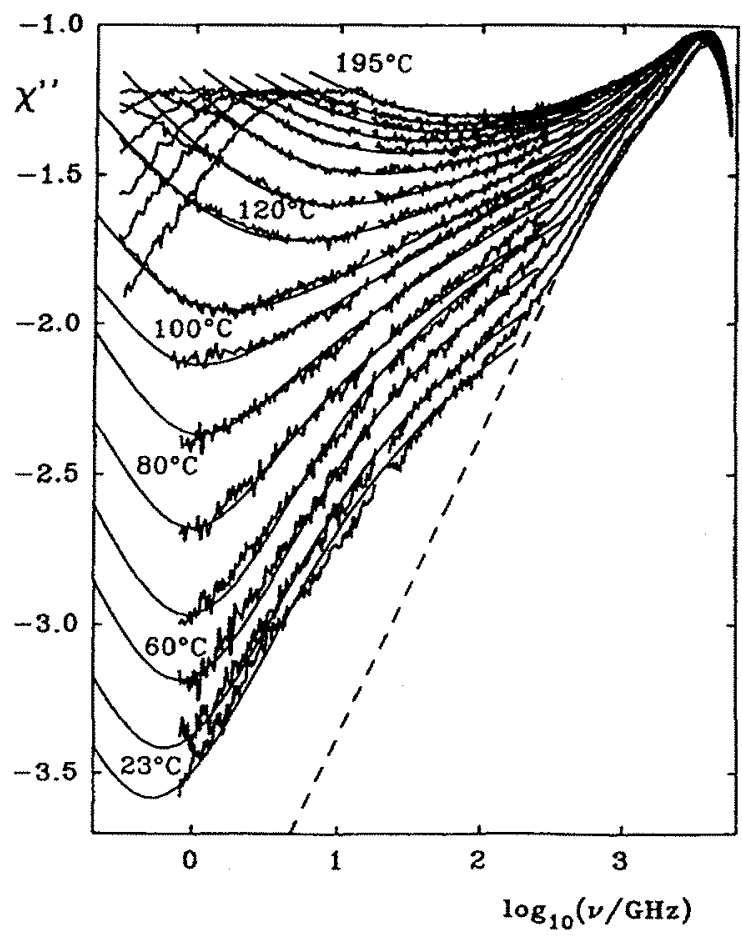

Susceptibility spectra $\chi^{\prime \prime}(\omega)$ obtained by depolarized light scattering in CKN (Li et al. 1992a). The solid lines are the MCT fits from Cummins et al. (1993) using the $\beta$-susceptibilities calculated from eqn. (2) with $\lambda=0.85$ and $\sigma$ and $\delta$ varied as shown in fig. 2. The critical amplitudes $h_{\mathrm{ls}}$ are shown in the inset of fig. 5. The dashed line is an upper limit to the Markovian result $\chi^{\prime \prime}(\omega) \propto \omega$ for the low-frequency wing of the microscopic excitations. 
Fig. 2

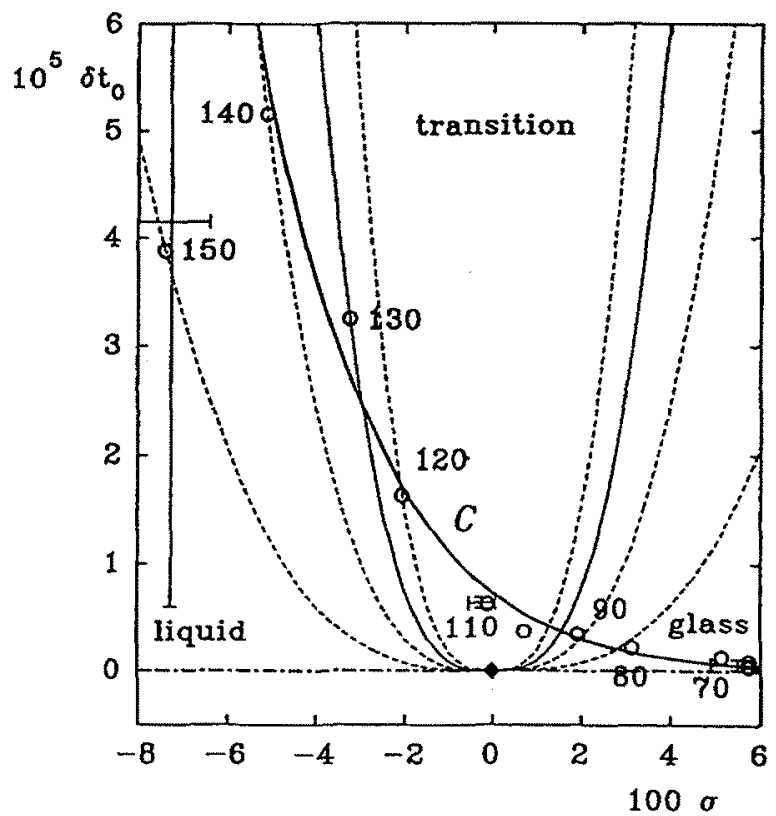

Control parameter space $\left(\sigma(T), \delta(T) t_{0}\right)$ (from Cummins et al. (1993)) explored by CKN for $23^{\circ} \mathrm{C} \leqslant T \leqslant 150^{\circ} \mathrm{C}$. For $160^{\circ} \mathrm{C} \leqslant T, \delta=0 \mathrm{can}$ be chosen. The approximate path $C$ (bold solid line) taken by CKN corresponds to the linear variation $\sigma(T)=0.52\left(T_{\mathrm{c}}-T\right) / T_{\mathrm{c}}$ and the Arrhenius variation $\delta(T) t_{0}=37568 \mathrm{exp}-E / R T$ with $T_{\mathrm{c}}=378.5 \mathrm{~K}$ and $E=16.8 \mathrm{kcal} \mathrm{mol}^{-1}$. The four generalized parabolas are scaling lines with $\sigma / \sigma_{0}= \pm 2.15, \pm 1.36, \pm 1$ and \pm 0.79 (listed by increasing steepness). They join in the glass-transition singularity at $(\sigma, \delta)=(0,0)$ marked by a diamond, which also separates the idealized liquid $(\sigma<0, \delta=0)$ from the idealized glass $(\sigma>0, \delta=0)$ scaling lines, both shown as chain curves.

The change in the spectral shape from a broad concave minimum at high temperatures to a steep minimum with a convex high-frequency side at low temperatures signals the asymmetry of the scaling law (2) for $\sigma<0$ and $\sigma>0$; see eqns. ( $4 a$ ) and (4b). From this one can estimate that $80^{\circ} \mathrm{C}<T_{\mathrm{c}}<120^{\circ} \mathrm{C}$. The detailed analysis presented in fig. 1 leads to $T_{\mathrm{c}}=105 \pm 5^{\circ} \mathrm{C}$. At $T=110^{\circ} \mathrm{C}$ (closest to $T_{\mathrm{c}}$ ) the critical power law $\chi^{\prime \prime} \propto \omega^{a}$ with $a=0.25$ can be observed for $c .1 .5$ decades, fixing $\lambda=0.85 \pm 0.02$. The control parameters $\left(\sigma(T), \delta(T) t_{0}\right)$ of this fit are shown in fig. 2. The only other fit parameter $h_{\mathrm{ss}}$ is shown in fig. $2(b)$ of Cummins et al. (1993) and will be discussed later in this paper (see inset of fig. 5). With changing temperature $\left(\sigma, \delta t_{0}\right)$ move along the path $C$ which corresponds to a linear variation in $\sigma(T)$ and an Arrhenius variation in $\delta(T) t_{0}$.

Figure 2 demonstrates how the conjecture of Goldstein (1969) is borne out in the scaling law (2). Goldstein reasoned that a change in transport mechanism from liquid- to solid-like should take place if, at some temperature $T^{*}$, the relaxation rates had slowed down some 3-4 decades, compared to the microscopic excitations of the liquid. Below T* he expected solid-like, i.e. thermally activated, processes, whereas above $T^{*}$ he suggested concepts of liquid dynamics (Goldstein 1969). In the idealized MCT the 'cage effect', well known from liquid dynamics (Hansen and McDonald 1986), is approximately and self-consistently contained and leads to the divergence of time scales such as $\omega_{\min } \propto \omega_{\sigma}$ for $T \backslash T_{\mathrm{c}}$. The extended MCT explains the cut off of these divergences $\left(\omega_{\min }\left(T_{c}\right) \approx \omega_{\delta\left(T_{\mathrm{c}}\right)}\right)$ due to the existence of further relaxation channels introducing one parameter $\delta(T)$ into eqn. (2). 
Fig. 3

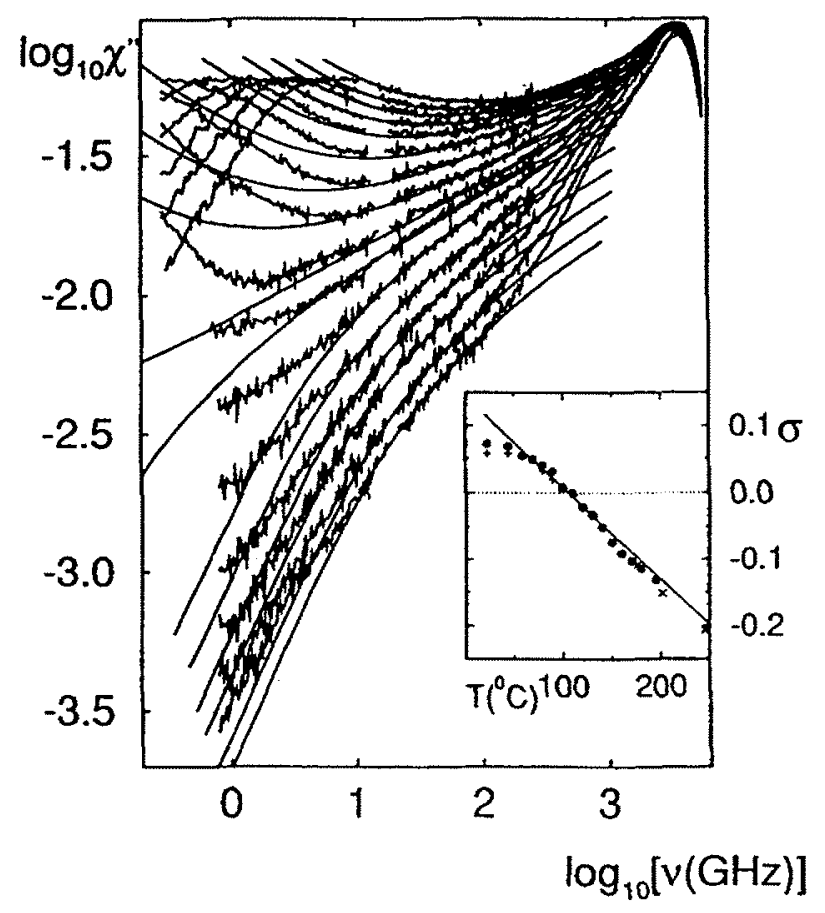

Idealized $(\delta=0)$ MCT fit to the light-scattering data from fig. 1. The exponent parameter $\lambda=0.85$ and the critical amplitudes (see inset of fig. 5) from the extended $(\delta>0)$ fit shown in fig. 1 are taken. The $\sigma(T)$ values of the fit from fig. 1 (fig. 3) are shown as plus (circle) in the inset (symbols coincide at most temperatures); results from the neutron spin-echo analysis $(x)$ and the linear fit $\sigma(T)=0.52\left(T_{c}-T\right) / T_{c}$ are also included.

Notice that in fig. 2 the scaling lines $\left(\delta t_{0}\right) \propto \sigma(T)^{1+2 a r 2 a}$ rise more steeply than the activated rate $\delta(T) \propto \exp (-E / k T)$ for $\sigma$ not too small. This explains the paradox that the activated rate $\delta$ can be neglected at higher temperatures.

The value of the idealized MCT as a zeroth approximation to the data can be judged from fig. 3. There, in contrast to fig. 1, only $\delta(T)=0$ is set for all temperatures. The deviations of the data from this $(\delta=0)$ fit appear at low frequencies for all except the highest temperatures; see Cummins et al. (1993) for a detailed discussion of how to anticipate these $(\delta>0)$ effects in such a fit. The $\sigma(T)$ values used in the fits of figs. 1 and 3 are shown $n$ the parameter plane in fig. 2 and in the inset of fig. 3 . Only at low temperatures were the $(\delta=0)$ fits improved by shifting $\sigma$ slightly. (These fits were already shown as $\chi^{\prime \prime}(\omega) / \sqrt{ } \omega$ in fig. 11 of Cummins et al. (1993).) The shown $\sigma$ values also differ little from the values of the fit shown in Li et al. (1992a).

The factorization property (1) can be tested directly in CKN using the extended neutron spin-echo data from Mezei (1991). As $G(t, \sigma(T), \delta(T))$ is known from $\chi^{\prime \prime}(\omega, \sigma(T), \delta(T)$ ), which was determined in the fits to the depolarized light-scattering data, only the $\alpha$-strength $f_{\mathrm{n}}^{\mathrm{c}}$ and the critical amplitude $h_{\mathrm{n}}$ can be varied in order to compare eqn. (1) to the neutron data. Figure 4 shows the resulting fits with $f_{\mathrm{n}}^{\mathrm{c}}=0.80$ and $h_{\mathrm{n}}(T)$ varying as shown in fig. 2 (b) of Cummins et al. (1993) and discussed later in this paper (see inset of fig. 5). The direct connection of light-scattering spectra of neutron spin-echo curves predicted by the factorization property (1) is non-trivial. For example, from $\chi^{\prime \prime}(\omega, \sigma(T), \delta(T))$ the time 
Fig. 4

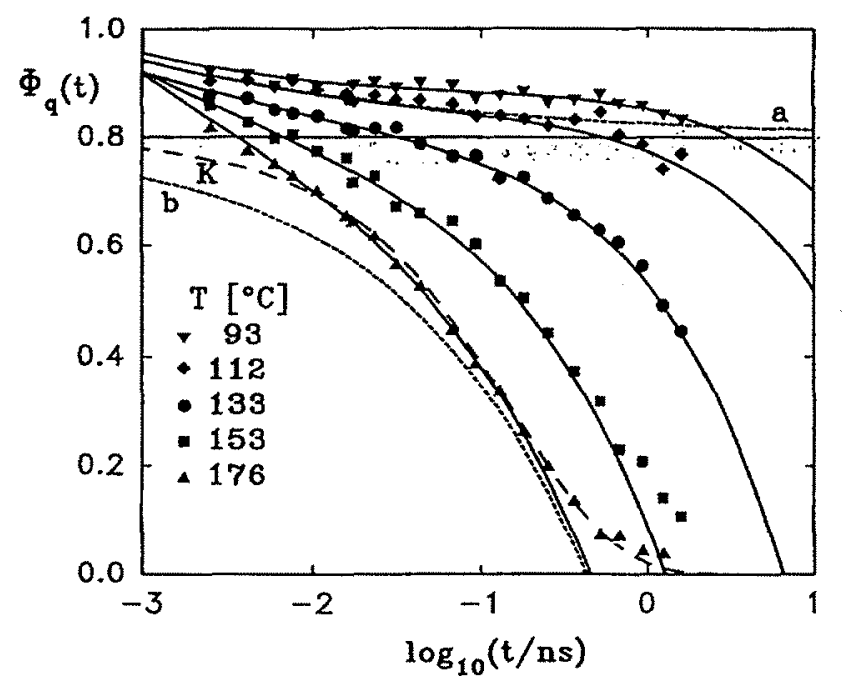

Neutron spin-echo data (from Mezei (1991)) with the extended MCT fits (from Cummins et al. (1993)) using the $\beta$-correlators $G(t, \sigma, \delta)$ predetermined from the light-scattering analysis. The horizontal line is the constant non-ergodicity parameter $f_{n}^{\mathfrak{c}}=0-80$. The critical amplitudes $h_{\mathrm{n}}$ are shown in the inset of fig. 5, The dashed line $a$ is the critical correlator corresponding to the fit at $T=112^{\circ} \mathrm{C}$ closest to $T_{\mathrm{c}}$. The dashed line $b$ is the von Schweidler asymptote of the $\delta=0$ master curve for $T=176^{\circ} \mathrm{C}$. The dashed curve marked $K$ is a Kohlrausch fit $A$ $\left.\exp -(t / \tau)_{\alpha}\right)^{\beta}$ to the $T=176^{\circ} \mathrm{C}$ data with fixed amplitude $A=f_{n}^{c}$ and fitted $\beta=0.71$ and $\log _{10} \tau_{d} / n s=-0.80$.

$t^{*}$, where $G\left(t^{*}, \sigma(T), \delta(T)\right)=0$, is fixed, because the microscopic matching time $t_{0}$ is the same for both quantities measured. The possibility of fixing $f_{n}^{c}=0.80 \pm 0.01$ for $90^{\circ} \mathrm{C} \leqslant T \leqslant 176^{\circ} \mathrm{C}$ and $h_{\mathrm{n}}=0.225$ for $90^{\circ} \mathrm{C} \leqslant T \leqslant 133^{\circ} \mathrm{C}$ in this analysis lends support to the factorization property (1).

It may be summarized that the MCT picture of the anomalous relaxation in an intermediate frequency window can be fitted to the data for the two experiments described. Generally the fit parameters exhibit temperature (in-)dependence as expected from the asymptotic results (1) and (2). However, the temperature dependence of the critical amplitudes, i.e. $h_{\mathrm{n}}$ above $T \geqslant 50^{\circ} \mathrm{C}$ and the drift of $h_{\mathrm{ls}}$ for all temperatures (also seen in depolarized light-scattering experiments in other systems ( $\mathrm{Li}$, Du, Sakai and Cummins 1992b, Du et al. 1994, Steffen, Patkowski, Gläser, Meier and Fischer 1994), indicates qualitative deviations of the data from the asymptotic results. Possible explanations for these effects are discussed in sections 4 and 5 .

\section{§. THE TEMPERATURE DEPENDENCE OF $h_{\mathrm{n}}$}

At low temperatures the slower $\alpha$-process is well separated from the $\beta$-process in fig. 4. The application of eqn. (1) with constant $h_{\mathrm{n}}$ also implies that $\alpha$-corrections are negligible. At higher temperatures, however, the separation shrinks down to $\tau / t_{\sigma} \approx 10$; for example, for $T=176^{\circ} \mathrm{C}, \log _{10}\left(t_{\sigma} / \mathrm{sec}\right) \approx-10.85$ and $\log _{10}\left(\tau \mathrm{s}^{-1}\right) \approx-9.84$ (estimating $\tau$ from $\left.\Phi(t=\tau)=f_{n}^{c} e^{-1}\right)$. Therefore the neutron data above $T \geqslant 153^{\circ} \mathrm{C}$ will be reconsidered in order to ascertain whether the $\alpha$-corrections to formula (1) can explain the temperature dependence of $h_{\mathrm{n}}$ at these larger separations from the critical point. A combined 
$\alpha-\beta$-analysis within the idealized MCT has to match the two asymptotic scaling laws (1) and (5) according to (Fuchs, Götze, Hildebrand and Latz 1992b)

$$
\Phi_{\mathrm{n}}(t)=f_{n}^{c} F_{\mathrm{n}}(t / \tau)+h_{\mathrm{n}}\left(G(t, \sigma, 0)+B \sigma^{1 / 2}\left(t / t_{\sigma}\right)^{b}\right),
$$

where $B \approx 1.85$ (Götze 1990). The $\alpha$-master function will be approximated by Kohlrausch function $F_{\mathrm{n}}(x)=\exp \left(-\left(x / x_{\mathrm{n}}\right)^{\beta_{\mathrm{n}}}\right)$ (Fuchs 1994). Neglecting thermally activated processes, which restricts this analysis to $T \geqslant 160^{\circ} \mathrm{C}$, the asymptotic result (8) requires $f_{\mathrm{n}}^{\mathrm{c}}, h_{\mathrm{n}}, x_{\mathrm{n}}$ and $\beta_{\mathrm{n}}$ to be temperature independent and $\tau$ abd $t_{\sigma}$ to be connected via eqn. $(5 b)$. As $f_{\mathrm{n}}^{c}=0.80$ and $h_{\mathrm{n}}=0.225$ were already determined from the fits in fig. 4 for low temperatures $T \leqslant 133^{\circ} \mathrm{C}$, only the two parameters $x_{n}=1.67$ and $\beta_{n}=0.63$ were adjusted in order to produce the fits of fig. 5. The only temperature-dependent fit parameter, $\sigma(T)$, was taken from the previous analysis where possible. Above the temperature range studied in the light-scattering experiment, $\sigma(T)$ follows via $(5 b)$ from $\tau(T)$. The new values are included in fig. 3. At $T=153^{\circ} \mathrm{C}$ the $(\delta>0)$ corrections to the $\beta$-correlator $G$ are neglected as eqn. (5) applies for $\delta=0$ only. Owing to the value $\sigma / \sigma_{0} \approx 2 \cdot 1$, this necessitates a change of $x_{\mathrm{n}}$ to $x_{\mathrm{n}}=0.81$ for this temperature. The $\alpha-\beta$-fits describe the data with constant critical amplitude $h_{\mathrm{n}}$ (see inset of fig. 5) up to $T \leqslant 246 \approx \mathrm{C}$, i.e. up to $140^{\circ} \mathrm{C}$ above $T_{\mathrm{c}}$.

One may conclude that the apparent temperature dependence of $h_{n}$ found in the earlier ( $\beta$-relaxation only) analysis (Cummins et al. 1993) was due to the neglect of $\alpha$-corrections to eqn. (1) if both processes for temperature far above $T_{\mathrm{c}}$ are not well separated.

\section{$\S 5$. THE TEMPERATURE DEPENDENCE OF $h_{\mathrm{ls}}$}

It is generally anticipated that the dipole-induced-dipole (DID) mechanism causes the depolarized light scattering observed in the discussed experiment [Li et al. 1992a]. It is well known that the DID mechanism exhibits a cancellation effect which leads to a strong suppression of the scattering intensity with increasing density (Thibeau, Gharbi, Le Duff and Sergiescu 1977). This has also been confirmed by recent molecular dynamics simulations (Watson and Madden 1993, Variyar, Noro and Kivelson 1993) and Monte Carlo calculations (Bykhovskii and Pick 1994). This cancellation effect is not contained in the often-used Stephen's approximation for the DID light spectrum, $I^{\mathrm{DID}}(\omega)=\int_{0}^{\infty} \mathrm{d} t \cos (\omega t) l^{\mathrm{DID}}(t)$, which results in (Stephen 1969):

$$
I^{\mathrm{DID} . \mathrm{s}}(t) \propto V \alpha^{4} \int \mathrm{d} k\left(V_{k}^{\mathrm{DID}, \mathrm{s}}\right)^{2} \Phi_{k}^{2}(t) \quad \text { and } \quad V_{k}^{\mathrm{DID}, \mathrm{s}}=\frac{n}{\left(15 \pi^{2}\right)^{1 / 2}} k S_{k} g(k a),
$$

where $\alpha$ is an effective polarizability, $\alpha=\partial \varepsilon / \partial n$, and $g(k a)$ is a cut-off function (normalized to $g(0=1)$ preventing self-polarization of particle with radius $a$. In eqn. (9) the total intensity $l^{\mathrm{DDD}}=l^{\mathrm{DID}}(t=0)$ and the critical amplitude $h_{15} \propto 2 \int \mathrm{d} k\left(V_{k}^{\mathrm{DID}, S}\right)^{2} f_{k}^{\mathrm{c}} h_{k}$ are expressed in terms of density correlation functions for different wave-vectors $k$. As the factorization property (1) is an exact result for the density correlators $\Phi_{\mathrm{q}}(t)$ within the full MCT equations of motion (Götze 1991) eqn. (9) implies that eqn. (1) should also hold for the DID light-scattering mechanism. The asymptotic results (1) and (2) then imply that the dominant temperature dependence of the spectra enters via the relevant control parameters $\left(\sigma(T), \delta(T) t_{0}\right)$, where $\sigma$ follows from the equilibrium structure factor $S_{\mathrm{q}}(T)$. In particular, the temperature dependence of the DID vertices $V_{k}^{\mathrm{DD}}$ should be small compared to the variation in $\sigma(T)$ which requires $h_{\mathrm{ls}} \approx h_{\mathrm{ls}}\left(T_{\mathrm{c}}\right)$. Temperature insensitivity of $h_{\mathrm{ls}}$ is not expected (Thibeau et al. 1977) and indeed not found in the data analysis of figs. 1 and 3 . It will be shown that the indicated problem arises from the oversimplified factorization performed in eqn. (9). It will be argued that this defect can be corrected in principle without ruining 
any of the previously studied implications of the MCT when applied to the light-scattering data.

It is well known from mode coupling theories for the dynamics of simple liquids that static correlations enter into the vertices of the corresponding mode coupling functional (Götze 1991). One may start from the following exact result for the anisotropic DID scattering of a system of spherical particles with point polarizability $\alpha_{i j}=\alpha_{0} \delta_{i j}$ (Fuchs and Latz 1991)

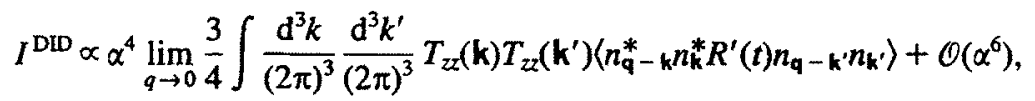

where $T_{z z}(q)$ is one component of the static DID propagator and $R^{\prime}(t)$ denotes a projected dynamics decoupled from hydrodynamic slow modes. The Stephens approximation (9) is obtained by simply factorizing the density pair-correlation function in eqn. (10). It leads to a total DID intensity $I^{\text {DID }}$ much too large for increasing density (Bykhovskii and Pick 1994). The static correlations of the DID mechanism are taken into account by a projection of the DID-propagated pair density on to density pairs and a following factorization of the dynamical pair density fluctuation function:

$$
I^{\mathrm{DID}} \propto V \alpha^{4} \frac{\mathrm{d}^{3} k}{(2 \pi)^{3}}\left(V_{k}^{\mathrm{DID}}\right)^{2} \Phi_{k}^{2}(t)
$$

with

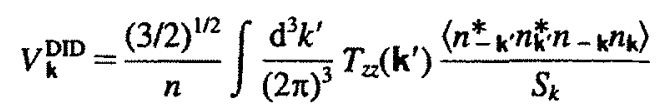

The result (11) now satisfies the condition that its total DID intensity is bounded by the exact result (10). Following the MCT scheme (Götze 1991) it is assumed that the missing intensity has to be added to (11) at high frequencies. In eqn. (11) the factorization of the density correlators (1) leads to the light-scattering critical amplitude $h_{\mathrm{ls}}$ :

$$
h_{1 \mathrm{~s}} \propto V \alpha^{4} \int \frac{\mathrm{d}^{3} k}{(2 \pi)^{3}}\left(V_{k}^{\mathrm{DD}}\right)^{2} 2 f_{k}^{\mathrm{c}} h_{k}
$$

which explicitly contains the static correlations of the DID mechanism in the vertex. From the molecular dynamics simulation (Watson and Madden 1993) in principle the $\sigma$-dependence of $h_{\mathrm{ls}}$ can be estimated. If one considers that $h_{\mathrm{ls}}$ is quadratic in the static DID correlation function determining the vertex $V_{k}^{\text {DID }}$, one can argue that the decrease in $h_{\mathrm{ls}}$ seen in different materials (Li et al. 1992a, b, Du et al. 1994, Steffen et al. 1994) will be contained in (12). Further computer simulation studies, however, will be necessary in order to check this expectation quantitatively. Nevertheless, from the above argument it is clear that the predictions of the MCT concerning the dynamics as described by the scaling law (2) can be tested by depolarized light-scattering experiments. The expected temperature dependence of $h_{\mathrm{ls}}$ has, however, to be taken into account in the analysis.

\section{§6. Conclusions}

The light-scattering experiments (Cummins et al. 1993, Du et al. 1994) study the dynamics within the intermediate or $\beta$-relaxation window where the universal MCT predictions contained in eqns. (1) and (2) can be tested most directly. Qualitative and quantitative agreement with the asymptotic scaling law (2) was obtained, except for the finding of a temperature-dependent critical amplitude $h_{\mathrm{ls}}$. The decrease of $h_{\mathrm{ls}}$ with increasing density or decreasing temperature may, however, be expected from the understood 
Fig. 5

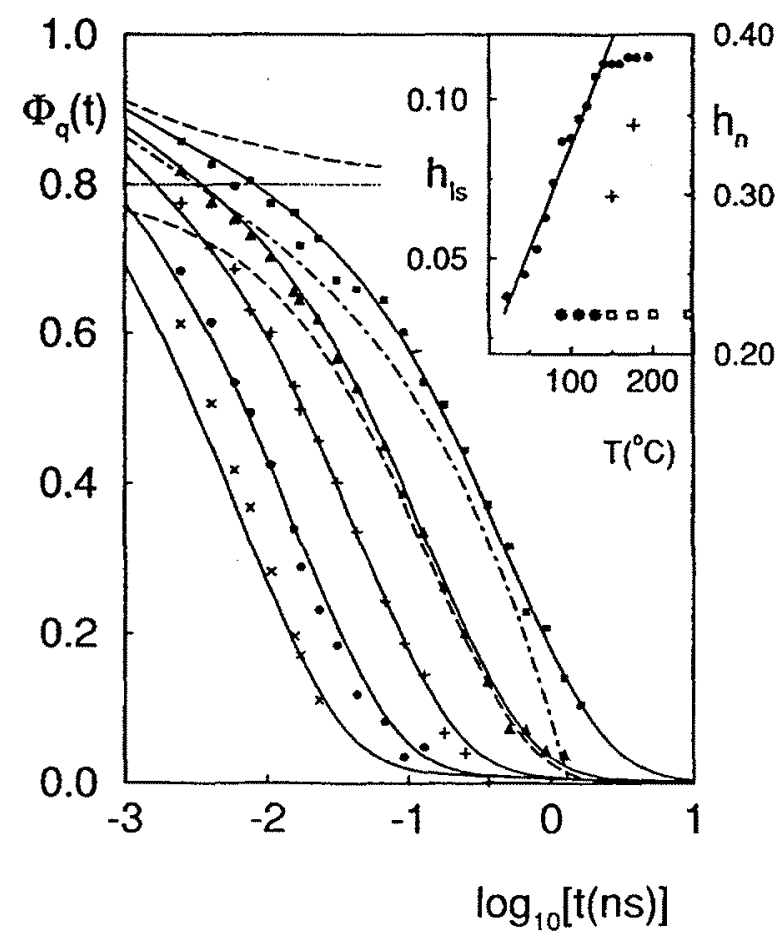

$\alpha-\beta$-fits of the idealized MCT to the neutron spin-echo data from Mezei (1991) for $T=153,176,202$, 246 and $287^{\circ} \mathrm{C}$ (from right to left). The solid lines are the $\alpha-\beta$-fits (eqn. (8)) with $\alpha$-strength $f_{\mathrm{n}}^{c}=0.80$ (dotted line), constant critical amplitude $h_{\mathrm{n}}=0.225$, Kohlrausch exponent $\beta_{\mathrm{n}}=0.63$ and $\alpha$-relaxation time $\tau_{n}=x_{n} \tau\left(x_{n}=1.67\right.$ and $\left.x_{n}\left(T=176^{\circ} \mathrm{C}\right)=0.81\right)$. The $\sigma$ values are included in the inset of fig. 3 . The lower long dashed curve shows the Kohlrausch law, the upper the $\beta$-corrections $G+B \sigma^{1 / 2}\left(t / t_{\sigma}\right)^{b}$ of eqn. (8), both adding up to the fit at $T=176^{\circ} \mathrm{C}$. The chain curve denotes the $\beta$-relaxation, $f_{n}^{c}+h_{n} G(t)$, at this temperature. The deviations of this $\beta$-only fit are conrected either by adding $\alpha$-relaxation as in this figure or by changing the critical amplitude to $h_{\mathrm{n}}\left(T=176^{\circ} \mathrm{C}\right)=0.34$ as in fig. 4 . The inset compares the temperature dependence of the critical amplitudes of the light $\left(h_{\mathrm{ts}}\right)$ and neutron $\left(h_{\mathrm{n}}\right)$ scattering analysis. The circles mark $h_{\mathrm{ls}}$, the crosses mark $h_{\mathrm{n}}$ of the $\beta$-analysis of fig. 4 and the squares mark the constant $h_{\mathrm{n}}$ values used in the combined $\alpha-\beta$-analysis of the higher-temperature data.

cancellation effect of the DID mechanism. This effect can easily be incorporated into the MCT expression for the light-scattering intensity (11). The factorization property (1) for the spectrum thus is recovered with $h_{1}$, however, showing the DID cancellation effect (12). This explanation will also carry over to other light-scattering mechanisms showing similar static correlation effects. In Cummins et al. [1993] a temperature dependence of the neutron scattering critical amplitude $h_{\mathrm{n}}$ was also reported, albeit a much smaller one than for $h_{\mathrm{ls}}$. It is therefore important to notice that the apparent temperature dependence of the neutron critical amplitude $h_{\mathrm{n}}$ was caused by the neglect of $\alpha$-corrections. Taking into account $\alpha$-corrections yields the perfect fit of fig. 5 with temperature-independent neutron critical amplitude $h_{\mathrm{n}}$.

The use of asymptotic scaling laws for temperature differences from the critical temperature $T_{\mathrm{c}}$ larger than $100^{\circ} \mathrm{C}$ may be unjustified. The possibility of meaningful quantitative fits, however, indicates that eqns. (1) and (2) qualitatively describe the dynamics within a rather large temperature region. 


\section{ACKNOWLEDGMENT}

This work was supported by the Deutsche Forschungsgemeinschaft under contract Go154/8-1.

\section{REFERENCES}

Barrat, J. L., Gö́tze, W., and Latz, A., 1989, J. Phys.: condens. Matter, 1, 7163.

BYkнovski, A. D., and Pick, R. M., 1994, J. chem. Phys. (to be published).

CuMmINS, H.Z., Du, W. M., FuChS, M., GớTZE, W., HILDEBRAND, S., LATZ, A., LI, G., and TAO, N. J., 1993, Phys. Rev. E, 47, 4223.

Du, W. M., Li, G., Cummins, H. Z., Fuchs, M., Tourouse, J., and KNauss, L. A., 1994, Phys. Rev. E, 49, 2192.

Fuchs, M., 1994, J. non-crystalline Solids, 172-174, 241.

Fuchs, M., and LaTZ, A., 1991, J. chem. Phys., 95, 7074.

Fuchs, M., Gơtze, W., Hindebrand, S., and LATZ, A., 1992a, J. Phys.: condens. Matter, 4, 7709;1992b, Z Phys. B, 87, 43.

Fuchs, M., Hofacker, I., and Latz, A., 1992c, Phys. Rev. A, 45, 898.

GoldSTEn, M., 1969, J. chem. Phys., 51, 3728.

GOTZE, W., and SJöGREN, L., 1987, Z. Phys. B, 65, 415; 1992, Rep. Prog. Phys., 55, 241.

GóTZE, W., 1990, J. Phys.: condens. Matter, 2, 8485; 1991, Liquids Freezing and Glass Transition, edited by J. P. Hansen, D. Levesque and J. Zinn-Justin (Amsterdam: North Holland), p. 763.

Götze, W., and SJögren, L., 1987, Z. Phys. B, 65, 415; 1992, Rep. Prog. Phys., 55, 241.

HANSEN, J.P., and MCDONALD, I. R., 1986, Theory of simple liquids, 2nd edition (London: Academic Press).

Li, G., Du, W. M., Chen, X. K., Cummins, H. Z., and TAo, N. J., 1992a, Phys. Rev. A, 45, 3867.

LI, G., Du, W. M., Sakai, A., and Cummins, H.Z., 1992b, Phys. Rev. A, 46, 3343.

MezeI, F., 1991, Ber. Bunsenges. Phys. Chem., 95, 1118; J. non-crystalline Solids, 131-133, 317.

NAGI, K. L., 1994, J. non-crystalline Solids (to be published).

SJÖGREN, L., 1990, Z. Phys. B, 79, 5.

StefFen, W.,PATKOWSKi, A., Gl.Äser, H., Meier, G., and FisCher, E. W., 1994, Phys. Rev. E, 49,2992

STEPHEN, M. J., 1969, Phys. Rev., 187, 247.

ThIBEAU, M., GharBi, Le DUfF, Y., and Sergiescu, V., 1977, J. Physique, 38, 641.

van Megen, W., and UnderwOod, S. M., 1993a, Phys. Rev. Lett., 70, 2766; 1993b, Phys. Rev. E, $47,248$.

Variyar, J. E., Noro, M., and Kuvelson, D., 1993, Molec. Phys., 79, 235.

Watson, S., and MADDEN, P. A., 1993, J. chem. Phys., 99, 6449. 